\title{
Symmetry of solutions to parabolic Monge-Ampère equations
}

Limei Dai*

\section{"Correspondence:}

limeidai@yahoo.com.cn

School of Mathematics and

Information Science, Weifang

University, Weifang, Shandong

261061, China

\begin{abstract}
In this paper, we study the parabolic Monge-Ampère equation$$
-u_{t} \operatorname{det}\left(D^{2} u\right)=f(t, u) \quad \text { in } \Omega \times(0, T] .
$$

Using the method of moving planes, we show that any parabolically convex solution is symmetric with respect to some hyperplane. We also give a counterexample in $\mathbb{R}^{n} \times(0, T]$ and an example in a cylinder to illustrate the results.

MSC: $35 \mathrm{~K} 96 ; 35 \mathrm{~B} 06$
\end{abstract}

Keywords: parabolic Monge-Ampère equations; symmetry; method of moving planes

\section{Introduction}

The Monge-Ampère equation has been of much importance in geometry, optics, stochastic theory, mass transfer problem, mathematical economics and mathematical finance theory. In optics, the reflector antenna system satisfies a partial differential equation of Monge-Ampère type. In $[1,2]$, Wang showed that the reflector antenna design problem was equivalent to an optimal transfer problem. An optimal transportation problem can be interpreted as providing a weak or generalized solution to the Monge-Ampère mapping problem [3]. More applications of the Monge-Ampère equation and the optimal transportation can be found in $[3,4]$. In the meantime, the Monge-Ampère equation turned out to be the prototype for a class of questions arising in differential geometry.

For the study of elliptic Monge-Ampère equations, we can refer to the classical papers [5-7] and the study of parabolic Monge-Ampère equations; see the references [811] etc. The parabolic Monge-Ampère equation $-u_{t} \operatorname{det}\left(D^{2} u\right)=f$ was first introduced by Krylov [12] together with the other parabolic versions of elliptic Monge-Ampère equations; see [8] for a complete description and related results. It is also relevant in the study of deformation of surfaces by Gauss-Kronecker curvature $[13,14]$ and in a maximum principle for parabolic equations [15]. Tso [15] pointed out that the parabolic equation $-u_{t} \operatorname{det}\left(D^{2} u\right)=f$ is the most appropriate parabolic version of the elliptic MongeAmpère equation $\operatorname{det}\left(D^{2} u\right)=f$ in the proof of Aleksandrov-Bakelman maximum principle of second-order parabolic equations. In this paper, we study the symmetry of solutions to the parabolic Monge-Ampère equation

$$
-u_{t} \operatorname{det}\left(D^{2} u\right)=f(t, u), \quad(x, t) \in Q,
$$

๑) 2013 Dai; licensee Springer. This is an Open Access article distributed under the terms of the Creative Commons Attribution License (http://creativecommons.org/licenses/by/2.0), which permits unrestricted use, distribution, and reproduction in any medium, provided the original work is properly cited. 


$$
\begin{aligned}
& u=0, \quad(x, t) \in S Q, \\
& u=u_{0}(x), \quad(x, t) \in B Q,
\end{aligned}
$$

where $D^{2} u$ is the Hessian matrix of $u$ in $x, Q=\Omega \times(0, T], \Omega$ is a bounded and convex open subset in $\mathbb{R}^{n}, S Q=\partial \Omega \times(0, T)$ denotes the side of $Q, B Q=\bar{\Omega} \times\{0\}$ denotes the bottom of $Q$, and $\partial_{p} Q=S Q \cup B Q$ denotes the parabolic boundary of $Q, f$ and $u_{0}$ are given functions.

There is vast literature on symmetry and monotonicity of positive solutions of elliptic equations. In 1979, Gidas et al. [16] first studied the symmetry of elliptic equations, and they proved that if $\Omega=\mathbb{R}^{n}$ or $\Omega$ is a smooth bounded domain in $\mathbb{R}^{n}$, convex in $x_{1}$ and symmetric with respect to the hyperplane $\left\{x \in \mathbb{R}^{n}: x_{1}=0\right\}$, then any positive solution of the Dirichlet problem

$$
\begin{aligned}
& \Delta u+f(u)=0, \quad x \in \Omega, \\
& u=0, \quad x \in \partial \Omega
\end{aligned}
$$

satisfies the following symmetry and monotonicity properties:

$$
\begin{aligned}
& u\left(-x_{1}, x_{2}, \ldots, x_{n}\right)=u\left(x_{1}, x_{2}, \ldots, x_{n}\right), \\
& u_{x_{1}}\left(x_{1}, x_{2}, \ldots, x_{n}\right)<0 \quad\left(x_{1}>0\right) .
\end{aligned}
$$

The basic technique they applied is the method of moving planes first introduced by Alexandrov [17] and then developed by Serrin [18]. Later the symmetry results of elliptic equations have been generalized and extended by many authors. Especially, Li [19] considered fully nonlinear elliptic equations on smooth domains, and Berestycki and Nirenberg [20] found a way to deal with general equations with nonsmooth domains using the maximum principles on domains with small measure. Recently, Zhang and Wang [21] investigated the symmetry of the elliptic Monge-Ampère equation $\operatorname{det}\left(D^{2} u\right)=e^{-u}$ and they got the following results.

Let $\Omega$ be a bounded convex domain in $\mathbb{R}^{n}$ with smooth boundary and symmetric with respect to the hyperplane $\left\{x \in \mathbb{R}^{n}: x_{1}=0\right\}$, then each solution of the Dirichlet problem

$$
\begin{aligned}
& \operatorname{det}\left(D^{2} u\right)=e^{-u}, \quad x \in \Omega, \\
& u=0, \quad x \in \partial \Omega
\end{aligned}
$$

has the above symmetry and monotonicity properties (1.4) and (1.5). Extensions in various directions including degenerate problems [22] or elliptic systems of equations [23] were studied by many authors.

For the symmetry results of parabolic equations on bounded and unbounded domains, the reader can be referred to $[16,24,25]$ and the references therein. In particular, when $Q=\Omega \times J, J=(0, T]$, Gidas et al. [16] studied parabolic equations $-u_{t}+\Delta u+f(t, r, u)=0$ and $-u_{t}+F\left(t, x, u, D u, D^{2} u\right)=0$, and they proved that parabolic equations possessed the same symmetry as the above elliptic equations. When $J=(0, \infty)$, Hess and Poláčik [25] 
first studied the asymptotic symmetry results for classical, bounded, positive solutions of the problem

$$
\begin{aligned}
& u_{t}-\Delta u=f(t, u), \quad(x, t) \in \Omega \times J, \\
& u=0, \quad(x, t) \in \partial \Omega \times J .
\end{aligned}
$$

The symmetry of general positive solutions of parabolic equations was investigated in [24, $26,27]$ and the references therein. A typical theorem of $J=\mathbb{R}$ is as follows.

Let $\Omega$ be convex and symmetric in $x_{1}$. If $u$ is a bounded positive solution of (1.6) and (1.7) with $J=\mathbb{R}$ satisfying

$$
\inf _{t \in \mathbb{R}} u(x, t)>0 \quad(x \in \Omega, t \in J)
$$

then $u$ has the symmetry and monotonicity properties for each $t \in \mathbb{R}$ :

$$
\begin{aligned}
& u\left(-x_{1}, x^{\prime}, t\right)=u\left(x_{1}, x^{\prime}, t\right) \quad\left(x=\left(x_{1}, x^{\prime}\right) \in \Omega, t \in \mathbb{R}\right), \\
& u_{x_{1}}(x, t)<0 \quad\left(x \in \Omega, x_{1}>0, t \in \mathbb{R}\right) .
\end{aligned}
$$

The result of $J=(0, \infty)$ is as follows.

Assume that $u$ is a bounded positive solution of (1.6) and (1.7) with $J=(0, \infty)$ such that for some sequence $t_{n} \rightarrow \infty$,

$$
\liminf _{n \rightarrow \infty} u\left(x, t_{n}\right)>0 \quad(x \in \Omega)
$$

Then $u$ is asymptotically symmetric in the sense that

$$
\begin{aligned}
& \lim _{t \rightarrow \infty}\left(u\left(-x_{1}, x^{\prime}, t\right)-u\left(x_{1}, x^{\prime}, t\right)\right)=0 \quad(x \in \Omega), \\
& \limsup _{t \rightarrow \infty} u_{x_{1}}(x, t) \leq 0 \quad\left(x \in \Omega, x_{1}>0\right) .
\end{aligned}
$$

In this paper, using the method of moving planes, we obtain the same symmetry of solutions to problem (1.1), (1.2) and (1.3) as elliptic equations.

\section{Maximum principles}

In this section, we prove some maximum principles. Let $\Omega$ be a bounded domain in $\mathbb{R}^{n}$, let $a^{i j}(x, t), b(x, t), c(x, t)$ be continuous functions in $\bar{Q}, Q=\Omega \times(0, T]$. Suppose that $b(x, t)<0$, $c(x, t)$ is bounded and there exist positive constants $\lambda_{0}$ and $\Lambda_{0}$ such that

$$
\lambda_{0}|\xi|^{2} \leq a^{i j}(x, t) \xi_{i} \xi_{j} \leq \Lambda_{0}|\xi|^{2}, \quad \forall \xi \in \mathbb{R}^{n}
$$

Here and in the sequel, we always denote

$$
D_{i}=\frac{\partial}{\partial x_{i}}, \quad D_{i j}=\frac{\partial^{2}}{\partial x_{i} \partial x_{j}} .
$$

We use the standard notation $C^{2 k, k}(Q)$ to denote the class of functions $u$ such that the derivatives $D_{x}^{i} D_{t}^{j} u$ are continuous in $Q$ for $i+2 j \leq 2 k$. 
Theorem 2.1 Let $\lambda(x, t)$ be a bounded continuous function on $\bar{Q}$, and let the positive function $\varphi \in C^{2,1}(\bar{Q})$ satisfy

$$
b(x, t) \varphi_{t}+a^{i j}(x, t) D_{i j} \varphi-\lambda(x, t) \varphi \leq 0 .
$$

Suppose that $u \in C^{2,1}(Q) \cap C^{0}(\bar{Q})$ satisfies

$$
\begin{aligned}
& b(x, t) u_{t}+a^{i j}(x, t) D_{i j} u-c(x, t) u \leq 0, \quad(x, t) \in Q, \\
& u \geq 0, \quad(x, t) \in \partial_{p} Q .
\end{aligned}
$$

If

$$
c(x, t)>\lambda(x, t), \quad(x, t) \in Q
$$

then $u \geq 0$ in $Q$.

Proof We argue by contradiction. Suppose there exists $(\bar{x}, \bar{t}) \in Q$ such that $u(\bar{x}, \bar{t})<0$. Let

$$
v(x, t)=\frac{u(x, t)}{\varphi(x, t)}, \quad(x, t) \in Q .
$$

Then $v(\bar{x}, \bar{t})<0$. Set $v\left(x_{0}, t_{0}\right)=\min _{\bar{Q}} v(x, t)$, then $x_{0} \in \Omega$ and $v\left(x_{0}, t_{0}\right)<0$. Since $v\left(\cdot, t_{0}\right)$ attains its minimum at $x_{0}$, we have $D v\left(x_{0}, t_{0}\right)=0, D^{2} v\left(x_{0}, t_{0}\right) \geq 0$. In addition, we have $v_{t}\left(x_{0}, t_{0}\right) \leq 0$. A direct calculation gives

$$
\begin{aligned}
& v_{t}=\frac{u_{t} \varphi-u \varphi_{t}}{\varphi^{2}}, \\
& D_{i j} \nu=\frac{1}{\varphi} D_{i j} u-\frac{u}{\varphi^{2}} D_{i j} \varphi-\frac{1}{\varphi} D_{i} v D_{j} \varphi-\frac{1}{\varphi} D_{j} v D_{i} \varphi .
\end{aligned}
$$

Taking into account $u\left(x_{0}, t_{0}\right)<0$, we have at $\left(x_{0}, t_{0}\right)$,

$$
\begin{aligned}
0 & \leq \varphi a^{i j} D_{i j} v=a^{i j} D_{i j} u-\frac{a^{i j} D_{i j} \varphi}{\varphi} u \\
& \leq a^{i j} D_{i j} u+\frac{u}{\varphi}\left(b \varphi_{t}-\lambda \varphi\right) \\
& \leq a^{i j} D_{i j} u+\frac{b}{\varphi} u_{t} \varphi-\lambda u \\
& =a^{i j} D_{i j} u+b u_{t}-\lambda u \\
& <a^{i j} D_{i j} u+b u_{t}-c u \\
& \leq 0 .
\end{aligned}
$$

This is a contradiction and thus completes the proof of Theorem 2.1.

Theorem 2.1 is also valid in unbounded domains if $u$ is nonnegative at infinity. Thus we have the following corollary. 
Corollary 2.2 Suppose that $\Omega$ is unbounded, $Q=\Omega \times(0, T]$. Besides the conditions of Theorem 2.1, we assume

$$
\liminf _{|x| \rightarrow \infty} u(x, t) \geq 0
$$

Then $u \geq 0$ in $Q$.

Proof Still consider $v(x, t)$ in the proof of Theorem 2.1. Condition (2.5) shows that the minimum of $v(x, t)$ cannot be achieved at infinity. The rest of the proof is the same as the proof of Theorem 2.1.

If $\Omega$ is a narrow region with width $l$,

$$
\Omega=\left\{x \in \mathbb{R}^{n} \mid 0<x_{1}<l\right\},
$$

then we have the following narrow region principle.

Corollary 2.3 (Narrow region principle) Suppose that $u \in C^{2,1}(Q) \cap C^{0}(\bar{Q})$ satisfies $(2.2)$ and (2.3). Let the width $l$ of $\Omega$ be sufficiently small. If on $\partial_{p} Q, u \geq 0$, then we have $u \geq 0$ in $Q$. If $\Omega$ is unbounded, and $\liminf _{|x| \rightarrow \infty} u(x, t) \geq 0$, then the conclusion is also true.

Proof Let $0<\varepsilon<l$,

$$
\varphi(x, t)=t+\sin \frac{x_{1}+\varepsilon}{l} .
$$

Then $\varphi$ is positive and

$$
\begin{aligned}
& \varphi_{t}=1, \\
& a^{i j} D_{i j} \varphi=-\left(\frac{1}{l}\right)^{2} a^{11} \varphi .
\end{aligned}
$$

Choose $\lambda(x, t)=-\lambda_{0} / l^{2}$. In virtue of the boundedness of $c(x, t)$, when $l$ is sufficiently small, we have $c(x, t)>\lambda(x, t)$, and thus

$$
\begin{aligned}
b \varphi_{t} & +a^{i j} D_{i j} \varphi-\lambda \varphi \\
& =b-\left(\frac{1}{l}\right)^{2} a^{11} \varphi-\left(-\frac{\lambda_{0}}{l^{2}}\right) \varphi \\
& =b-\left(\frac{1}{l}\right)^{2} a^{11} \varphi+\frac{\lambda_{0}}{l^{2}} \varphi \\
& \leq b<0 .
\end{aligned}
$$

From Theorem 2.1, we have $u \geq 0$. 


\section{Main results}

In this section, we prove that the solutions of (1.1), (1.2) and (1.3) are symmetric by the method of moving planes.

Definition 3.1 A function $u(x, t): Q \rightarrow \mathbb{R}$ is called parabolically convex if it is continuous, convex in $x$ and decreasing in $t$.

Suppose that the following conditions hold.

(A) $f_{u}(t, u) / f(t, u)$ is bounded in $[0, T] \times \mathbb{R}$.

(B) $\partial u_{0} / \partial x_{1}<0$ and

$$
u_{0}(x) \leq u_{0}\left(x^{\lambda}\right), \quad x \in \Omega^{\lambda}
$$

where $x^{\lambda}=\left(2 \lambda-x_{1}, x_{2}, \ldots, x_{n}\right), \Omega^{\lambda}=\Omega \cap\left\{x \in \Omega: x_{1} \leq \lambda\right\}(\lambda<0)$.

Theorem 3.1 Let $\Omega$ be a strictly convex domain in $\mathbb{R}^{n}$ and symmetric with respect to the plane $\left\{x \in \Omega: x_{1}=0\right\}, Q=\Omega \times(0, T]$. Assume that conditions (A) and (B) hold and $u \in C^{2,1}(Q) \cap C^{0}(\bar{Q})$ is any parabolically convex solution of (1.1), (1.2) and (1.3). Then $u\left(x_{1}, x^{\prime}, t\right)=u\left(-x_{1}, x^{\prime}, t\right)$, where $(x, t)=\left(x_{1}, x^{\prime}, t\right) \in \mathbb{R}^{n+1}$, and when $x_{1} \geq 0, \partial u(x, t) / \partial x_{1} \leq 0$.

Proof Let in $\Omega^{\lambda} \times(0, T], u^{\lambda}(x, t)=u\left(x^{\lambda}, t\right)$, that is,

$$
u^{\lambda}\left(x_{1}, x_{2}, \ldots, x_{n}, t\right)=u\left(2 \lambda-x_{1}, x_{2}, \ldots, x_{n}, t\right), \quad(x, t) \in \Omega^{\lambda} \times(0, T] .
$$

Then

$$
D^{2} u^{\lambda}\left(x_{1}, x_{2}, \ldots, x_{n}, t\right)=P^{T} D^{2} u\left(2 \lambda-x_{1}, x_{2}, \ldots, x_{n}, t\right) P
$$

where $P=\operatorname{diag}(-1,1, \ldots, 1)$. Therefore,

$$
\begin{aligned}
-u_{t}^{\lambda} \operatorname{det}\left(D^{2} u^{\lambda}\right) & =-u_{t}\left(2 \lambda-x_{1}, x_{2}, \ldots, x_{n}, t\right) \operatorname{det}\left(D^{2} u\left(2 \lambda-x_{1}, x_{2}, \ldots, x_{n}, t\right)\right) \\
& =f\left(t, u\left(2 \lambda-x_{1}, x_{2}, \ldots, x_{n}, t\right)\right) \\
& =f\left(t, u^{\lambda}\right) .
\end{aligned}
$$

We rewrite (3.2) in the form

$$
\log \left(-u_{t}^{\lambda}\right)+\log \left(\operatorname{det}\left(D^{2} u^{\lambda}\right)\right)=\log f\left(t, u^{\lambda}\right)
$$

On the other hand, from (1.1), we have

$$
\log \left(-u_{t}\right)+\log \left(\operatorname{det}\left(D^{2} u\right)\right)=\log f(t, u)
$$

According to (3.3) and (3.4), we have

$$
\log \left(-u_{t}\right)-\log \left(-u_{t}^{\lambda}\right)+\log \left(\operatorname{det}\left(D^{2} u\right)\right)-\log \left(\operatorname{det}\left(D^{2} u^{\lambda}\right)\right)=\log f(t, u)-\log f\left(t, u^{\lambda}\right) .
$$


Therefore

$$
\begin{aligned}
& \int_{0}^{1} \frac{d}{d s} \log \left(-s u_{t}-(1-s) u_{t}^{\lambda}\right) d s+\int_{0}^{1} \frac{d}{d s} \log \operatorname{det}\left(s D^{2} u+(1-s) D^{2} u^{\lambda}\right) d s \\
& \quad=\int_{0}^{1} \frac{d}{d s} \log f\left(t, s u+(1-s) u^{\lambda}\right) d s .
\end{aligned}
$$

As a result, we have

$$
b(x, t)\left(u-u^{\lambda}\right)_{t}+a^{i j}(x, t)\left(u-u^{\lambda}\right)_{i j}-c(x, t)\left(u-u^{\lambda}\right)=0, \quad(x, t) \in \Omega^{\lambda} \times(0, T],
$$

where

$$
\begin{aligned}
& b(x, t)=\int_{0}^{1} \frac{d s}{s u_{t}+(1-s) u_{t}^{\lambda}}, \\
& a^{i j}(x, t)=\int_{0}^{1} g_{s}^{i j} d s, \\
& c(x, t)=\int_{0}^{1} \frac{f_{u}}{f}\left(t, s u+(1-s) u^{\lambda}\right) d s,
\end{aligned}
$$

$g_{s}^{i j}$ is the inverse matrix of $s D^{2} u+(1-s) D^{2} u^{\lambda}$. Then $b(x, t)<0, c(x, t)$ is bounded and by the a priori estimate [9] we know there exist positive constants $\lambda_{0}$ and $\Lambda_{0}$ such that

$$
\lambda_{0}|\xi|^{2} \leq a^{i j} \xi_{i} \xi_{j} \leq \Lambda_{0}|\xi|^{2}, \quad \forall \xi \in \mathbb{R}^{n}
$$

Let

$$
w^{\lambda}=u-u^{\lambda}
$$

then from (3.5),

$$
b(x, t) w_{t}^{\lambda}+a^{i j}(x, t) w_{i j}^{\lambda}-c(x, t) w^{\lambda}=0, \quad(x, t) \in \Omega^{\lambda} \times(0, T] .
$$

Clearly,

$$
w^{\lambda}(x, t)=0, \quad x \in \partial \Omega^{\lambda} \cap\left\{x_{1}=\lambda\right\}, 0<t \leq T .
$$

Because the image of $\partial \Omega \cap \partial \Omega^{\lambda}$ about the plane $\left\{x_{1}=\lambda\right\}$ lies in $\Omega$, according to the maximum principle of parabolic Monge-Ampère equations,

$$
u^{\lambda}(x, t) \leq 0, \quad \forall x \in \partial \Omega \cap \partial \Omega^{\lambda} .
$$

Thus

$$
w^{\lambda}(x, t)=u-u^{\lambda}=0-u^{\lambda} \geq 0, \quad x \in \partial \Omega \cap \partial \Omega^{\lambda}, 0<t \leq T .
$$


On the other hand, from (3.1),

$$
w^{\lambda}(x, 0)=u_{0}(x)-u_{0}\left(x^{\lambda}\right) \geq 0, \quad x \in \Omega^{\lambda} .
$$

From Corollary 2.3, when the width of $\Omega^{\lambda}$ is sufficiently small, $w^{\lambda}(x, t) \geq 0,(x, t) \in \Omega^{\lambda} \times$ $(0, T]$.

Now we start to move the plane to its right limit. Define

$$
\Lambda=\sup \left\{\lambda<0 \mid w^{\lambda}(x, t) \geq 0, x \in \Omega^{\lambda}, 0<t \leq T\right\} .
$$

We claim that

$$
\Lambda=0 .
$$

Otherwise, we will show that the plane can be further moved to the right by a small distance, and this would contradict with the definition of $\Lambda$.

In fact, if $\Lambda<0$, then the image of $\partial \Omega \cap \partial \Omega^{\Lambda}$ under the reflection about $\left\{x_{1}=\Lambda\right\}$ lies inside $\Omega$. According to the strong maximum principle of parabolic Monge-Ampère equations, for $x \in \Omega, u^{\Lambda}<0$. Therefore, for $x \in \partial \Omega^{\Lambda} \cap \partial \Omega$, we have $w^{\Lambda}>0$. On the other hand, by the definition of $\Lambda$, we have for $x \in \Omega^{\Lambda}, w^{\Lambda} \geq 0$. So, from the strong maximum principle [28] of linear parabolic equations and (3.6), we have for $(x, t) \in \Omega^{\Lambda} \times(0, T]$,

$$
w^{\Lambda}(x, t)>0 .
$$

Let $d_{0}$ be the maximum width of narrow regions so that we can apply the narrow region principle. Choose a small positive constant $\delta$ such that $\Lambda+\delta<0, \delta \leq d_{0} / 2-\Lambda$. We consider the function $w^{\Lambda+\delta}(x, t)$ on the narrow region

$$
\Sigma^{\Lambda+\delta} \times(0, T]=\left(\Omega^{\Lambda+\delta} \cap\left\{x_{1}>\Lambda-\frac{d_{0}}{2}\right\}\right) \times(0, T] .
$$

Then $w^{\Lambda+\delta}(x, t)$ satisfies

$$
b(x, t) w_{t}^{\Lambda+\delta}+a^{i j}(x, t) D_{i j} w^{\Lambda+\delta}-c(x, t) w^{\Lambda+\delta}=0, \quad(x, t) \in \Sigma^{\Lambda+\delta} \times(0, T] .
$$

Now we prove the boundary condition

$$
w^{\Lambda+\delta}(x, t) \geq 0, \quad(x, t) \in \partial_{p}\left(\Sigma^{\Lambda+\delta} \times(0, T]\right)
$$

Similar to boundary conditions (3.7), (3.8) and (3.9), boundary condition (3.12) is satisfied for $x \in \partial \Sigma^{\Lambda+\delta} \cap \partial \Omega, x \in \partial \Sigma^{\Lambda+\delta} \cap\left\{x_{1}=\Lambda+\delta\right\}$ and for $t=0$. In order to prove (3.12) is satisfied for $x \in \partial \Sigma^{\Lambda+\delta} \cap\left\{x_{1}=\Lambda-d_{0} / 2\right\}$, we apply the continuity argument. By (3.10) and the fact that $\left(\Lambda-d_{0} / 2, x_{2}, \ldots, x_{n}\right)$ is inside $\Omega^{\Lambda}$, there exists a positive constant $c_{0}$ such that

$$
w^{\Lambda}\left(\Lambda-\frac{d_{0}}{2}, x_{2}, \ldots, x_{n}, t\right) \geq c_{0}
$$


Because $w^{\lambda}$ is continuous in $\lambda$, then for small $\delta$, we still have

$$
w^{\Lambda+\delta}\left(\Lambda-\frac{d_{0}}{2}, x_{2}, \ldots, x_{n}, t\right) \geq 0 .
$$

Therefore boundary condition (3.12) holds for small $\delta$. From Corollary 2.3, we have

$$
w^{\Lambda+\delta}(x, t) \geq 0, \quad x \in \Sigma^{\Lambda+\delta}, 0<t \leq T .
$$

Combining (3.10) and the fact that $w^{\lambda}$ is continuous for $\lambda$, we know that $w^{\Lambda+\delta}(x, t) \geq 0$ for $x \in \Omega^{\Lambda}$ when $\delta$ is small. Then from (3.13), we know that

$$
w^{\Lambda+\delta}(x, t) \geq 0, \quad x \in \Omega^{\Lambda+\delta}, 0<t \leq T .
$$

This contradicts with the definition of $\Lambda$, and so $\Lambda=0$.

As a result, $w^{0}(x, t) \geq 0$ for $x \in \Omega^{0}$, which means that as $x_{1}<0$,

$$
u\left(x_{1}, x_{2}, \ldots, x_{n}, t\right) \geq u\left(-x_{1}, x_{2}, \ldots, x_{n}, t\right) .
$$

Since $\Omega$ is symmetric about the plane $\left\{x_{1}=0\right\}$, then for $x_{1} \geq 0, u\left(-x_{1}, x_{2}, \ldots, x_{n}, t\right)$ also satisfies (1.1). Thus we can move the plane from the right towards the left and get the reverse inequality. Therefore

$$
\begin{aligned}
& \partial u(x, t) / \partial x_{1} \leq 0, \quad x_{1} \geq 0, \\
& u\left(x_{1}, x_{2}, \ldots, x_{n}, t\right)=u\left(-x_{1}, x_{2}, \ldots, x_{n}, t\right) .
\end{aligned}
$$

Equation (3.14) means that $u$ is symmetric about the plane $\left\{x_{1}=0\right\}$. Theorem 3.1 is proved.

If we put the $x_{1}$ axis in any direction, from Theorem 3.1, we have the following.

Corollary 3.2 If $\Omega$ is a ball, $Q=\Omega \times(0, T]$, then any parabolically convex solution $u \in$ $C^{2,1}(\bar{Q})$ of (1.1), (1.2) and (1.3) is radially symmetric about the origin.

Remark 3.1 Solutions of $(1.1)$ in $\mathbb{R}^{n} \times(0, T]$ may not be radially symmetric. For example,

$$
-u_{t} \operatorname{det}\left(D^{2} u\right)=e^{-u}, \quad(x, t) \in \mathbb{R}^{n} \times(0, T]
$$

has a non-radially symmetric solution. In fact, we know that $f(x)=2 \log \left(1+e^{\sqrt{2} x}\right)-\sqrt{2} x-$ $\log 4(x>0)$ satisfies $f^{\prime \prime}=e^{-f}$ in $\mathbb{R}^{1}$, and $f(x)=f(-x), x<0$. Define

$$
u(x, t)=\log (T-t)+f\left(x_{1}\right)+f\left(x_{2}\right)+\cdots+f\left(x_{n}\right)
$$

then $u$ is a solution of (3.15) but not radially symmetric. 
We conclude this paper with a brief examination of Theorem 3.1. Let $B=B_{1}(0)$ be the unit ball in $\mathbb{R}^{n}$, and let radially symmetric function $u_{0}(x)=u_{0}(r), r=|x|$ satisfy

$$
\begin{aligned}
& \frac{u_{0}(r)\left(u_{0}^{\prime}(r)\right)^{n-1} u_{0}^{\prime \prime}(r)}{r^{n-1}}=-1, \quad 0<r<1, \\
& u_{0}(1)=u_{0}^{\prime}(0)=0 .
\end{aligned}
$$

Example 3.1 Let $u_{0}$ satisfy (3.16) and (3.17). Then any solution of

$$
\begin{aligned}
& -u_{t} \operatorname{det}\left(D^{2} u\right)=1, \quad(x, t) \in B \times(0, T], \\
& u=0, \quad(x, t) \in \partial B \times(0, T), \\
& u=u_{0}, \quad(x, t) \in \bar{B} \times\{0\}
\end{aligned}
$$

is of the form

$$
u=-[(n+1) t+1]^{\frac{1}{n+1}} u_{0}(r),
$$

where $r=|x|$.

Proof According to Corollary 3.2, the solution is symmetric. Let

$$
u(x, t)=u(r, t), \quad r=|x| .
$$

Then

$$
\begin{aligned}
& u_{i}=\frac{\partial u(r, t)}{\partial r} \frac{x_{i}}{r} \\
& u_{i j}=\frac{\partial^{2} u(r, t)}{\partial r^{2}} \frac{x_{i} x_{j}}{r^{2}}+\frac{\partial u(r, t)}{\partial r}\left(\frac{\delta_{i j}}{r}-\frac{x_{i} x_{j}}{r^{3}}\right), \\
& \operatorname{det}\left(D^{2} u\right)=\left(\frac{\partial u / \partial r}{r}\right)^{n-1} \frac{\partial^{2} u}{\partial r^{2}} .
\end{aligned}
$$

Therefore (3.18) is

$$
-\frac{\partial u}{\partial t}\left(\frac{\partial u / \partial r}{r}\right)^{n-1} \frac{\partial^{2} u}{\partial r^{2}}=1
$$

We seek the solution of the form

$$
u(r, t)=T(t) u_{0}(r) .
$$

Then

$$
-u_{0}(r) T^{\prime}(t) \frac{\left(u_{0}^{\prime}(r) T(t)\right)^{n-1}}{r^{n-1}} u_{0}^{\prime \prime}(r) T(t)=1
$$

That is,

$$
\frac{u_{0}(r)\left(u_{0}^{\prime}(r)\right)^{n-1} u_{0}^{\prime \prime}(r)}{r^{n-1}}=-\frac{1}{T^{\prime}(t)(T(t))^{n}} .
$$


Therefore

$$
T^{\prime}(t)(T(t))^{n}=1
$$

By (3.20), we know that

$$
T(0)=1 \text {. }
$$

From (3.24) and (3.25), we have

$$
T(t)=[(n+1) t+1]^{\frac{1}{n+1}} .
$$

As a result,

$$
u(r, t)=-[(n+1) t+1]^{\frac{1}{n+1}} u_{0}(r) .
$$

From the maximum principle, we know that the solution of (3.18)-(3.20) is unique. Thus any solution of (3.18), (3.19) and (3.20) is of the form of (3.21).

\section{Competing interests}

The author declares that they have no competing interests.

\section{Acknowledgements}

The research was supported by NNSFC (11201343), Shandong Province Young and Middle-Aged Scientists Research Awards Fund (BS2011SF025), Shandong Province Science and Technology Development Project (2011YD16002).

Received: 3 April 2013 Accepted: 5 August 2013 Published: 20 August 2013

\section{References}

1. Wang, XJ: On the design of a reflector antenna. Inverse Probl. 12, 351-375 (1996)

2. Wang, XJ: On the design of a reflector antenna II. Calc. Var. Partial Differ. Equ. 20, 329-341 (2004)

3. Evans, LC, Gangbo, W: Differential Equations Methods for the Monge-Kantorovich Mass Transfer Problem. Mem. Amer. Math. Soc., vol. 137 (1999)

4. Gangbo, W, McCann, RJ: The geometry of optimal transportation. Acta Math. 177, 113-161 (1996)

5. Caffarelli, L, Nirenberg, L, Spruck, J: The Dirichlet problem for nonlinear second-order elliptic equations. I. Monge-Ampère equation. Commun. Pure Appl. Math. 37, 369-402 (1984)

6. Gutiérrez, CE: The Monge-Ampère Equation. Birkhäuser, Basel (2001)

7. Gilbarg, D, Trudinger, NS: Elliptic Partial Differential Equations of Second Order, 2nd edn. Springer, Berlin (1983)

8. Lieberman, GM: Second Order Parabolic Differential Equations. World Scientific, River Edge (1996)

9. Wang, GL: The first boundary value problem for parabolic Monge-Ampère equation. Northeast. Math. J. 3, 463-478 (1987)

10. Wang, RH, Wang, GL: On the existence, uniqueness and regularity of viscosity solution for the first initial boundary value problem to parabolic Monge-Ampère equations. Northeast. Math. J. 8, 417-446 (1992)

11. Xiong, JG, Bao, JG: On Jörgens, Calabi, and Pogorelov type theorem and isolated singularities of parabolic Monge-Ampère equations. J. Differ. Equ. 250, 367-385 (2011)

12. Krylov, NV: Sequences of convex functions, and estimates of the maximum of the solution of a parabolic equation. Sib. Mat. Zh. 17, 290-303 (1976) (in Russian)

13. Firey, WJ: Shapes of worn stones. Mathematika 21, 1-11 (1974)

14. Tso, K: Deforming a hypersurface by its Gauss-Kronecker curvature. Commun. Pure Appl. Math. 38, 867-882 (1985)

15. Tso, K: On an Aleksandrov-Bakelman type maximum principle for second-order parabolic equations. Commun. Partial Differ. Equ. 10, 543-553 (1985)

16. Gidas, B, Ni, WM, Nirenberg, L: Symmetry and related properties via the maximum principle. Commun. Math. Phys. 68, 209-243 (1979)

17. Alexandrov, AD: A characteristic property of spheres. Ann. Mat. Pura Appl. 58, 303-315 (1962)

18. Serrin, J: A symmetry problem in potential theory. Arch. Ration. Mech. Anal. 43, 304-318 (1971)

19. Li, C: Monotonicity and symmetry of solutions of fully nonlinear elliptic equations on unbounded domains. Commun. Partial Differ. Equ. 16, 585-615 (1991)

20. Berestycki, H, Nirenberg, L: On the method of moving planes and the sliding method. Bol. Soc. Bras. Mat. 22, 1-37 (1991) 
21. Zhang, ZT, Wang, KL: Existence and non-existence of solutions for a class of Monge-Ampère equations. J. Differ. Equ. $246,2849-2875(2009)$

22. Serrin, J, Zou, H: Symmetry of ground states of quasilinear elliptic equations. Arch. Ration. Mech. Anal. 148, 265-290 (1999)

23. Busca, J, Sirakov, B: Symmetry results for semilinear elliptic systems in the whole space. J. Differ. Equ. 163, 41-56 (2000)

24. Földes, J: On symmetry properties of parabolic equations in bounded domains. J. Differ. Equ. 250, 4236-4261 (2011)

25. Hess, P, Poláčik, P: Symmetry and convergence properties for non-negative solutions of nonautonomous reaction-diffusion problems. Proc. R. Soc. Edinb., Sect. A 124, 573-587 (1994)

26. Babin, AV: Symmetrization properties of parabolic equations in symmetric domains. J. Differ. Equ. 123, 122-152 (1995)

27. Babin, AV, Sell, GR: Attractors of non-autonomous parabolic equations and their symmetry properties. J. Differ. Equ. $160,1-50(2000)$

28. Protter, MH, Weinberger, HF: Maximum Principles in Differential Equations. Springer, New York (1984)

doi:10.1186/1687-2770-2013-185

Cite this article as: Dai: Symmetry of solutions to parabolic Monge-Ampère equations. Boundary Value Problems 2013 2013:185

Submit your manuscript to a SpringerOpen ${ }^{\odot}$ journal and benefit from:

- Convenient online submission

- Rigorous peer review

- Immediate publication on acceptance

- Open access: articles freely available online

- High visibility within the field

- Retaining the copyright to your article

Submit your next manuscript at $\gg$ springeropen.com 